\title{
Formation of Radiatively cooled, Supersonically Rotating, Plasma Flows in Z-pinch Experiments; towards the development of an experimental platform to study accretion disk physics in the laboratory.
}

\author{
M. J. Bennett ${ }^{a, *}$, S. V. Lebedeva,*, G. N. Hall ${ }^{\mathrm{a}, \mathrm{b}}$, L. Suttle ${ }^{\mathrm{a}}$, G. Burdiak ${ }^{\mathrm{a}}$, F. Suzuki-Vidal ${ }^{\mathrm{a}}$, J. Hare ${ }^{\mathrm{a}}$, G. Swadling ${ }^{\mathrm{a}}$, S. \\ Patankar $^{a}$, M. Bocchi ${ }^{a}$, J. P. Chittenden ${ }^{a}$, R. Smith ${ }^{\mathrm{a}}$, A. Frank ${ }^{\mathrm{c}}$, E. Blackman ${ }^{\mathrm{c}}$, R. P. Drake ${ }^{\mathrm{d}}$, A. Ciardi ${ }^{\mathrm{e}, \mathrm{f}}$ \\ ${ }^{a}$ Blackett Laboratory, Imperial College London, UK \\ ${ }^{b}$ Lawrence Livermore National Laboratory, USA \\ ${ }^{c}$ Department of Physics \& Astronomy, University of Rochester, USA \\ ${ }^{d}$ Department of Atmospheric, Oceanic and Space Science, University of Michigan, USA \\ e Sorbonne Universités, UPMC Univ. Paris 6, UMR 8112, LERMA, F-75005, Paris, France \\ ${ }^{f}$ LERMA, Observatoire de Paris, PSL Research University, CNRS, UMR 8112, F-75014, Paris France
}

\begin{abstract}
We present data from the first z-pinch experiments aiming to simulate aspects of accretion disk physics in the laboratory. Using off axis ablation flows from a wire array z-pinch we demonstrate the formation of a supersonically $(M \sim 2)$ rotating hollow plasma cylinder of height $\sim 4 \mathrm{~mm}$ and radius $2 \mathrm{~mm}$. Using a combination of diagnostics we measure the rotation speed $\left(\sim 60 \mathrm{kms}^{-1}\right)$, electron density $\left(10^{19} \mathrm{~cm}^{-3}\right)$, ion temperature $\left(T_{i} \sim 60 \mathrm{eV}\right)$ and the product of electron temperature and average ionisation $\left(Z T_{e} \sim 150\right.$ to $\left.200 \mathrm{eV}\right)$. Using these parameters we calculate the Reynolds number for the plasma on the order $10^{5}$ and magnetic Reynolds number as 10 - 100. The plasma flow is maintained for $150 \mathrm{~ns}$, corresponding to one rotation period, which should allow for studying fast instabilities which develop on this time-scale.
\end{abstract}

\section{Introduction}

Accretion disks are found in many astrophysical systems ranging from proto-stars to Active Galactic Nuclei. To accrete mass to a central object requires transfer of matter from greater to lesser radii and either a radially outward transfer of angular momentum or its vertical removal by outflows. Radial angular momentum transport in disks relies on an anomalous, turbulent viscosity whose origin is presently not fully understood. One of the most promising mechanisms to generate the needed turbulence in magnetized disks is the magneto-rotational instability [1].

Laboratory experiments offer the opportunity to study scaled plasma dynamics relevant to astrophysical accretion disks. Computational studies have already shown that scalable accretion disk plasmas should be achievable on Z-pinch experiments $[2,3]$. The ability to controllably introduce rotation to a z-pinch system was first shown by Ampleford et al [4] for rotating jets. Building on this experience a rotating plasma experiment was designed which would be complimentary to laser driven work first proposed by Ryutov [5]; work on this laser driven focus is currently being undertaken by Drake et al [R. P. Drake, private correspondence].

\footnotetext{
*Please address correspondance to either author

Email addresses: m.bennett11@imperial.ac.uk (M. J. Bennett), s.lebedev@imperial.ac.uk (S. V. Lebedev)
}

\section{Experiment}

Experiments are done on the Imperial College MAGPIE facility (250 ns, 1.4 MA) [6] using ablation flows from a cylindrical wire array z-pinch. Current passing through the wires $\left(J_{z}\right)$ creates a global $B_{\theta}$ which produces radial flows convergent on the array axis due to a Lorentz force $F_{r}=J_{z} \times B_{\theta}$. The converging flows are maintained in a quasi-steady state for the duration of the current pulse or until a significant fraction of the wire mass has been ablated; this results in the accumulation of mass on the axis.

Angular momentum, with respect to the symmetry axis, can be introduced to the flow by the addition of a radial field $B_{r}$ at the wires $[4,2]$ which gives a Lorentz force $F_{\theta}=$ $J_{z} \times B_{r}$. The combination of $F_{r}$ and $F_{\theta}$ produces "off axis" flows at an angle $\theta$ with respect to the radial direction $\hat{r}$. The convergence of these flows produces a rotating plasma structure (Figure 1b). If the magnetic field components $B_{r}$ and $B_{\theta}$ are solely produced by the generator current then the ratio $v_{r} / v_{\theta}$ will be approximately constant in time.

To produce a radial magnetic field, $B_{r}$, the wire array connects two oppositely wound coils above and below the wires (Figure 1a). As current flows through the array, and the coils, oppositely aligned magnetic fields are created which form a magnetic cusp in the plane of the wires; therefore producing a radially directed $B$ field at the wires. The coils are made from $1 \mathrm{~mm}$ diameter stainless steel and are manufactured with highly accurate/reproducible 
degrees of rotation (number of turns); examples used for this experiment are $2 \pi / 16$ and $2 \pi / 8$ rotated coils. Each coil is $28 \mathrm{~mm}$ long with an inner diameter of $16 \mathrm{~mm}$; consequently this sets the diameter of the wire array. The number of rods that make up the coils equals the number of wires used in the array. The array wires are $4 \mathrm{~mm}$ long of typically 40 $\mu \mathrm{m}$ aluminium with each wire connecting to an individual rod from the top and bottom coils.

To sustain the rotating plasma as long as possible the experiments are designed to maximise the duration of the ablation phase of the wires.The duration of the ablation phase can be controlled by the choice of wire diameter and the number of wires; however changing the number of wires also affects the uniformity of the converging flows. Variation of the twist angle of the cusp coils allows for control of the level of angular momentum injected by affecting the angle $\theta$ of the ablation flows. The end of the ablation phase and subsequent implosion of the array, which disrupts the rotating plasma, are not studied in this work.

The number of wires used ( 8 or 16) in the experiments are selected to improve the diagnostic access via 16 side on diagnostic ports on the MAGPIE facility. We study the general evolution of the plasma using (end-on) imaging on a fast 12-frame optical camera or a 4 -frame MCP XUV camera. The 12-frame camera allows us to observe the thermal emissions of the plasma in 5 ns exposures with variable inter-frame timings; typically 20 ns. The XUV camera has a 3 ns exposure with 30 ns inter-frame which is used to image the hottest regions of the plasma which are emitting soft X-rays; the minimum photon energy detectable is $30 \mathrm{eV}$. The use of end-on diagnostics provides information about the azimuthal structure of the ablation flows and the rotating plasma that is formed in the centre of the array. The electron density distribution can be studied using end-on laser interferometry at $532 \mathrm{~nm}$ and 355 $\mathrm{nm}$ (for further detail on our interferometry diagnostic see [7]). Interferograms allow for measurements of the flow density however the density gradients in the accumulated plasma are too great for most of the experiment and therefore only a lower bound can be provided.

A key parameter of interest is the velocity of the rotating plasma which is directly measured using a Thomson scattering diagnostic. The set-up used is similar to that discussed in [8] and uses a $532 \mathrm{~nm}$ ( $\sim 3 \mathrm{~J}, 8 \mathrm{~ns}$ width) laser to measure light scattered from the plasma in a collective regime (scattering parameter $\alpha \geq 1$ ). Measurements of the velocity profile were obtained as follows.

The Thomson scattering laser propagates through the mid plane between the wires as shown in Figure 1a and 1b. Scattered light is collected in the same plane at \pm $90^{\circ}$ to the direction of the beam by imaging the scattering volume onto two optical fibre bundles. The geometry for this is shown in Figure 1c. The bundles contain 14 individual fibres with $100 \mu \mathrm{m}$ diameter at a $250 \mu \mathrm{m}$ separation. The spectra of the scattered light is then recorded using an imaging Andor Shamrock 500 spectrometer with a time gated (4 ns) Andor iStar ICCD camera. The spectral res-

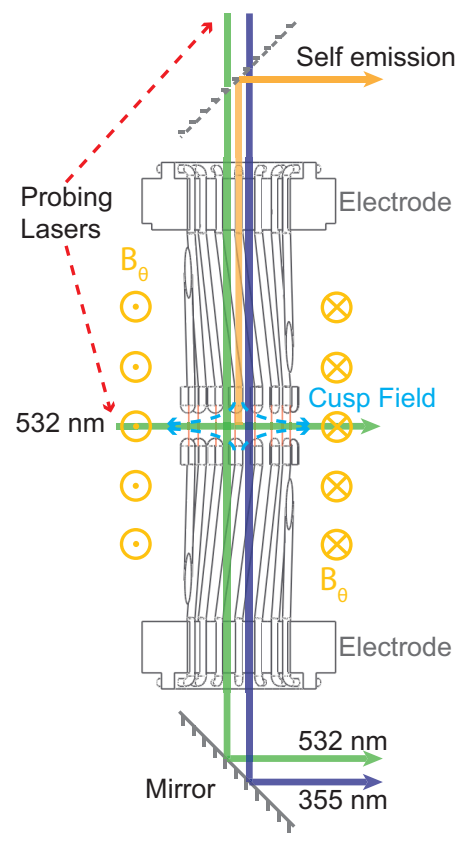

(a) Diagnostic access

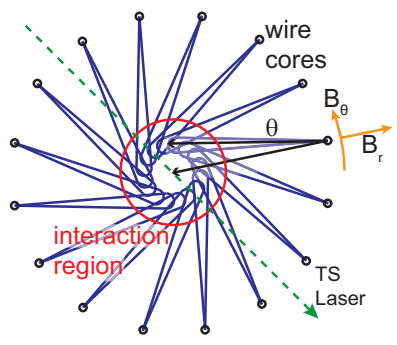

(b) Flow angle

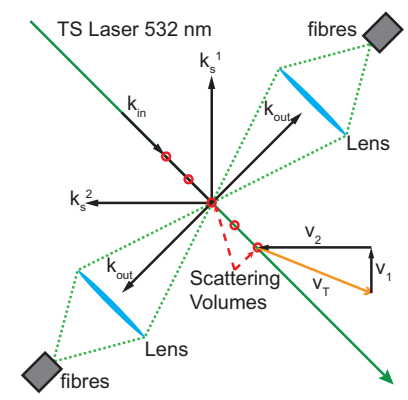

(c) TS Diagnostic
Figure 1: (a) shows a side-on cut through the array overlaid with magnetic fields created by the current and the paths for diagnostic access. (b) End-on schematic demonstrating the "off axis" convergence of the ablation flows and laser probing between the wires. (c) Schematic for Thomson scattering diagnostic showing the geometry relevant to equation (1) .

olution is $0.25 \AA$.

The detected light will be Doppler shifted by the bulk motion of the plasma such that

$$
\Delta \omega=\mathbf{k}_{\mathbf{s}} \cdot \mathbf{v}
$$

where $\mathbf{v}$ is the velocity, $\Delta \omega=\omega_{i}-\omega_{s}$ is the frequency shift, and $\mathbf{k}_{\mathbf{s}}=\mathbf{k}_{\mathbf{o}}-\mathbf{k}_{\mathbf{i}}$ is the scattering vector (Figure $1 \mathrm{c}$ ). By collecting the scattered light at $\pm 90^{\circ}$, and measuring the corresponding Doppler shifts, we can determine projections of the the plasma velocity in the two orthogonal directions ( $k_{s}^{1}$ and $k_{s}^{2}$ in Figure 1c) using (1). With orthogonal component velocities it is then straightforward to construct the final velocity vector and magnitude. The shape of spectrum provides further opportunity to analyse the plasma temperature and ionisation; for full details see $[8,9]$.

\section{Results}

\subsection{Formation of the Rotating Plasma}

In the initial phases of the experiment the ablation flows from the wires stagnate off-axis and due to their angular momentum form a hollow cylindrical structure. In the rotating plasma the centrifugal force $v_{\theta}^{2} / R$, along with the internal pressure of the plasma, is balanced by the ram pressure $\rho v_{r}^{2}$ from the ablation flows. This force balance sustains the geometry and rotation for the duration of the experiment. 


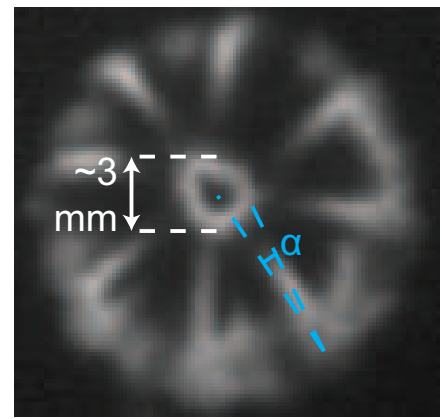

(a)

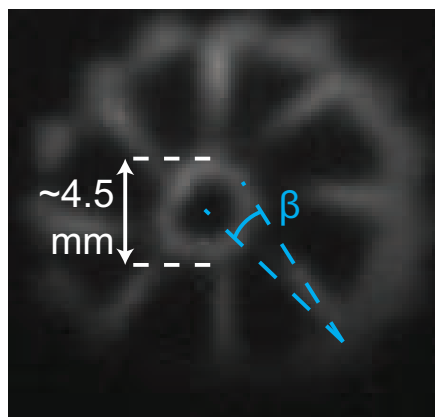

(b)

Figure 2: 12 frame images from (a) $2 \pi / 16$ and (b) $2 \pi / 8$ coil arrays to show the effect varying the twist angle has on the experiment. The shown angles are $\alpha \sim 9^{\circ}$ and $\beta \sim 14^{\circ}$. The reduction in emission along the flow lines is due to a combination of radiative cooling and flow divergence decreasing the density.

Figure 2 demonstrates the structure of the plasma flows and the effect the strength of $B_{r}$ has on the flows and the formation of the rotating plasma. In both images we observe the formation of a hollow cylinder about the axis, having reasonable symmetry, with its size determined by the degree of twist in the coils. More turns corresponds to a stronger cusp magnetic field and therefore greater $B_{r}$. As $B_{\theta}$ is determined by the radius of the array, which is the same in all experiments, this results in a larger flow angle. Figure $2 \mathrm{a}$ shows the flow angle for a $2 \pi / 16$ twist array as $\alpha \sim 9^{\circ}$ while $2 \mathrm{~b}$ shows $\beta \sim 14^{\circ}$ for a $2 \pi / 8$ twist; from these we observe rings with diameters $3 \mathrm{~mm}$ and $4.5 \mathrm{~mm}$ respectively. The axial thickness of the plasma is $4 \mathrm{~mm}$, i.e. the same as the wire length, meaning that for both arrays the height $H \gg R$ giving a cylindrical structure.

In general we find that for a $2 \pi / 16$ coil array with 8 wires the flow angle is $8-10^{\circ}$ whereas for 16 wires the angle is $6-7^{\circ}$. We would expect that the angle remains constant regardless of the choice of wire number ( 8 or 16) because the total force from the magnetic fields is determined only by the ratio of $B_{r}$ and $B_{\theta}$. The observed difference is likely to be a combination of localised current affects, as the plasma corona through which current flows are obviously much closer in a 16 wire array, and the divergence of the plasma causing interactions close to the wires [7].

Detailed temporal evolution of the rotating plasma from a 16 wire $2 \pi / 16$ array is shown in Figure 3 . The rotating flow appears to form, or is at least first viewable, at 150 ns after current start whereby we observe a ring like flow centred on the axis with $r=1.6 \mathrm{~mm}$ and thickness approximately $0.5 \mathrm{~mm}$. By considering the expected time for ablation start, $\sim 80 \mathrm{~ns}$, and the time of flight of plasma from the wires to the cylinder, $\sim 40 \mathrm{~ns}$ (at $\sim 1.4 \times 10^{7} \mathrm{cms}^{-1}$, see [8] and 3.3), we can calculate the expected formation time as $120 \mathrm{~ns}$. This agrees reasonably well with the observed time and very well with GORGON simulations of this experiment [2] (also $120 \mathrm{~ns}$ ); therefore this is the formation time that will be used in further analysis. From the
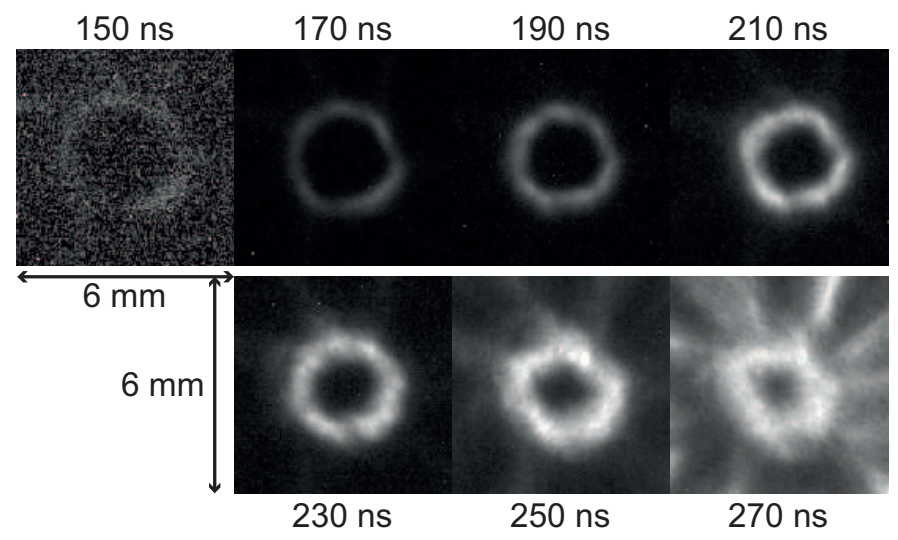

Figure 3: 12 frame evolution of $16 \mathrm{x} 40 \mu \mathrm{m} \mathrm{Al}$ array starting at 150 ns after the current pulse and ending at $270 \mathrm{~ns}$. The brightness levels on the first image have been adjusted in order to more clearly show the plasma ring. The thickness of the plasma in the $\mathrm{z}$ direction is 4 mm.

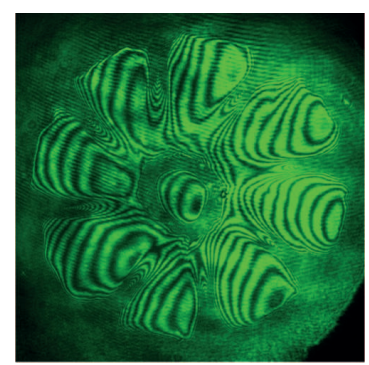

(a) Raw Interferogram

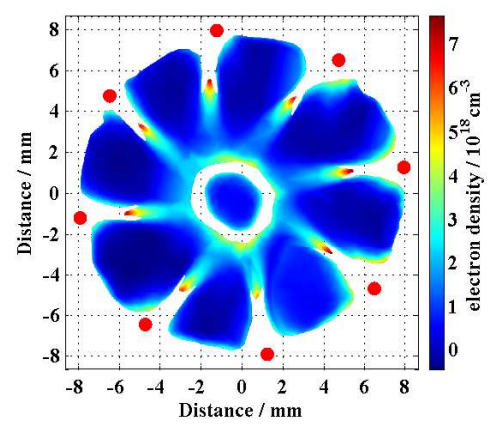

(b) Electron density map
Figure 4: (a) End-on interferogram from $532 \mathrm{~nm}$ laser at $200 \mathrm{~ns}$. (b) Electron density map of (a). Masked regions indicate where the density gradients are too high to observe fringes.

full images, similar to Figure 2 but for 16 wires, we estimate the flow width as $0.5 \mathrm{~mm}$; this is roughly the same as the initial ring thickness. After initial formation the rotating flow remains approximately circular for the next 100 ns until $t \simeq 250 \mathrm{~ns}$. Reasons for the flow becoming more irregular after this time are currently being investigated.

\subsection{Plasma Density}

Laser probing can be used to provide further information on the plasma structures and in-particular the density. The electron density $n_{e}$ is measured from end-on interferograms similar to Figure 4a by tracing the position of the fringes and applying the techniques described in detail by [7]. The interferogram shown in Figure $4 \mathrm{a}$ is for an 8 wire array at $200 \mathrm{~ns}$. The electron density in the off-axis ablation flows are on average a few $10^{18} \mathrm{~cm}^{-3}$ with highs closest to the wire of up to $7 \times 10^{18} \mathrm{~cm}^{-3}$ and $\sim 3 \times 10^{18} \mathrm{~cm}^{-3}$ just outside the rotating flow. Inside the flow cannot be probed due to its large density gradients. Nevertheless we can use the surrounding data to put a lower bound on the electron density in the flow of the order $10^{19} \mathrm{~cm}^{-3}$. 


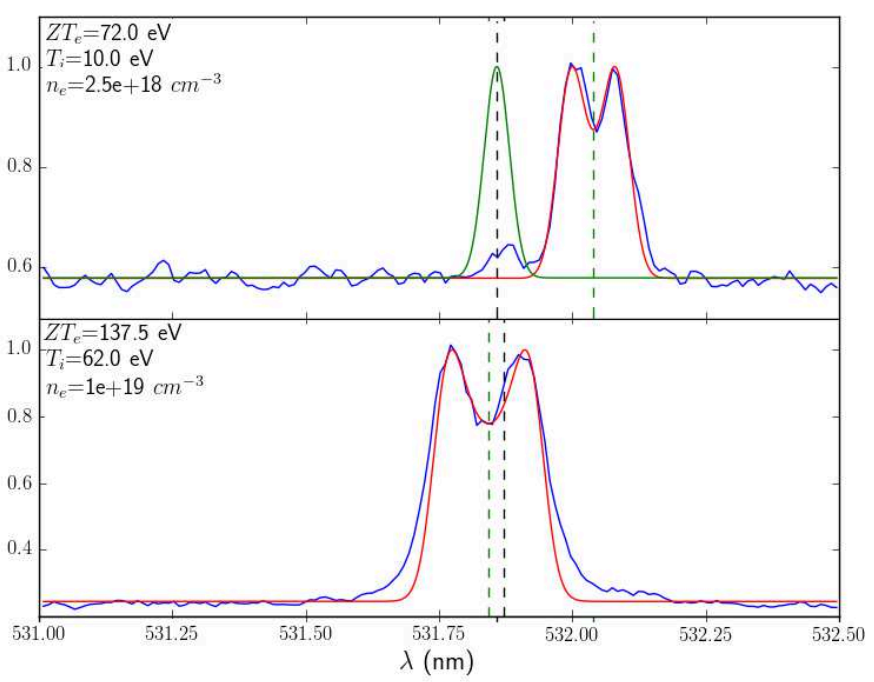

Figure 5: Thomson Scattered spectra from plasma at $r=2.25 \mathrm{~mm}$, the ablation flow (top), and $r=1.3 \mathrm{~mm}$, within the rotating flow (bottom). $r$ is defined from the array axis. The blue profiles are the line average of the imaged spectra. Black dotted lines show laser wavelength, i.e. $\Delta \lambda=0$, whilst green dotted lines show central Doppler shifted frequency of the spectra. The red lines show a spectral fit to the data using the equations for collective plasma scattering in [9] whilst also taking into account the instrument function (green Gaussian on top image).

\subsection{Velocity and Temperature}

Both the optical emission and interferometry show the formation of a hollow, cylindrical, structure that is consistent with the plasma rotating. This is confirmed by using a Thomson scattering technique to directly measure the plasma velocity and rotation profile. Figure 5 shows the Thomson scattered spectra at radial positions within the ablation flow $(r=2.25 \mathrm{~mm})$ and the rotating plasma $(r=1.3 \mathrm{~mm})$. Each shows both broadening of the spectra and a shift from $\lambda_{0}$. The shape provides information about the plasma temperatures whilst the shift provides measurement of the flow velocity.

The first step in the analysis is to determine the central wavelength, $\lambda$, of the scatter from which $\omega_{s}$ and $k_{s}$ can be calculated. Equation (1) is then used to calculate a corresponding velocity for the observed shift. For each scattering volume we measure $\Delta \lambda$ at $\pm 90^{\circ}$ observation angle giving us orthogonal components of velocity (along the projections $k_{s}^{1}$ and $k_{s}^{2}$, Figure 1c) from which we calculate $v_{r}$ and $v_{\theta}$. Results from this analysis for a single experiment at $t=190 \mathrm{~ns}$ are shown in Figure 6.

The $v_{r}$ component of the velocities, along the beam line, lie in the range 20 to $40 \mathrm{kms}^{-1}$ when measured within the rotating flow. Similar Thomson measurements performed at $r$ outside the ring radius show an inward radial flow $v_{r} \gtrsim 100 \mathrm{kms}^{-1}$ (also [8]) demonstrating a significant change in the radial velocity profile across the rotating cylinder boundary.

A firm confirmation for rotation is provided in Figure 6 which shows that the component of velocity perpendicular

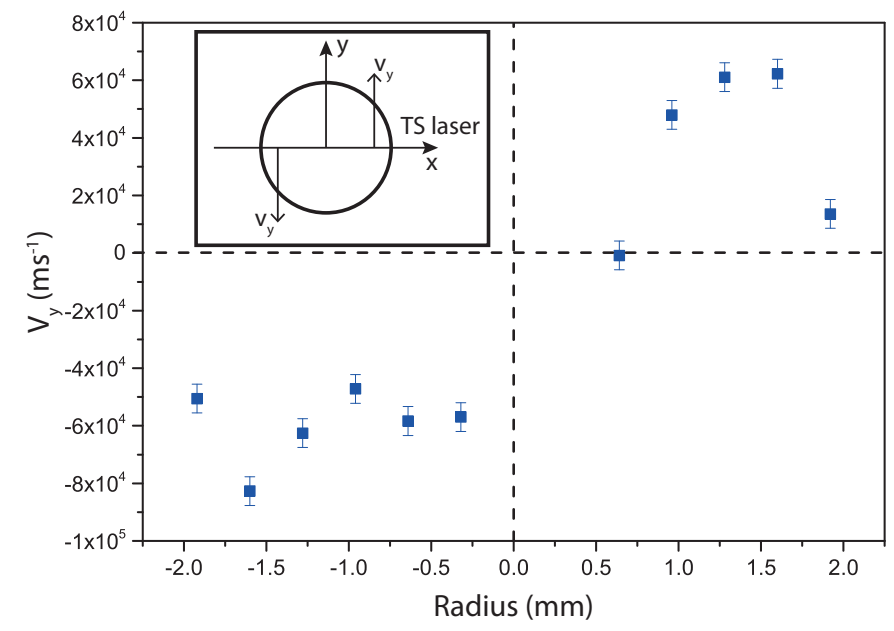

Figure 6: Thomson data showing the rotation velocity $\left(v_{y}\right)$ on an 8 wire array $190 \mathrm{~ns}$ after current start. The radial axis in all cases is measured along the beam line $(\mathrm{x})$ with 0 being the centre of the array. The resolution of the spectrometer is $0.25 \AA$ with corresponding velocity error $\pm 5 \mathrm{kms}^{-1}$. The inset shows the definition of $\mathrm{x}$ and $\mathrm{y}$ with respect to the Thomson laser.

to the beam, $v_{y}$ (see 6 ), is oppositely directed either side of the axis. This corresponds to a coherent rotation velocity $v_{\theta}$ with a mean of $60 \mathrm{kms}^{-1}$. At the mean velocity an element of plasma at a distance of $1.5 \mathrm{~mm}$ from the axis would take $\sim 160$ ns to complete a full rotation. Based on the time-scales discussed previously it is therefore possible for an element present at formation to complete approximately one rotation. The number of rotations is an important experimental parameter as it is related to the growth factor of shear flow instabilities $[5,10]$ that can potentially develop within the flow. The investigation into such instabilities is to be the focus of future work.

The second part of the Thomson scattered data analysis is to study the shape of the scattered spectra to infer the plasma temperature and ionisation. The line profiles shown in Figure 5 compare spectra from scatter within the rotating flow (bottom) and from the ablation flow just outside it (top). The fits shown are for theoretical form factors of the ion feature of the spectra calculated using equations from [9]. The form of the scattered spectrum is highly dependent on the ratio of $Z T_{e} / T_{i}$ and the characteristic ion-acoustic double peak structure, as seen in Figure 5, corresponds to $Z T_{e} / T_{i}>1$. The increase in peak separation between the ablation and rotating plasma is due to an increase in the parameter $Z T_{e}$ indicating a hotter and more ionised plasma. In the ablation flows the fitted form factors correspond to $Z T_{e} \sim 70 \mathrm{eV}$ and $T_{i} \simeq 10$ $\mathrm{eV}$. These parameters change drastically for the rotating plasma whereby $Z T_{e}$ increases to the range 150 to $200 \mathrm{eV}$ and $T_{i}$, although varying somewhat, is typically of the order of $60 \mathrm{eV}$. The observed increase in the parameters suggest there is shock heating at the rotating cylinder boundary allowing for a change in state of the plasma. Using the above information we can make estimates for the Mach 
numbers for the ablation flow and the rotating plasma. For the ablation flow we find $M=6$ to 8 , which agrees well with previous results obtained on standard cylindrical wire arrays [7], and for the rotating flow $M \sim 2$ which puts it firmly in a supersonic regime.

\section{Conclusions and Astrophysical Relevance}

In this work we have presented results from the first experiments aimed at modelling aspects of accretion disk physics on a z-pinch device. The experiments show the formation of a rotating hollow cylinder of height $4 \mathrm{~mm}$ and radius $2 \mathrm{~mm}$. The rotation is sustained for approximately $150 \mathrm{~ns}$, corresponding to one full rotation of the plasma, by the ram pressure from the ablation flows. During this time the plasma electron density is of the order $10^{19} \mathrm{~cm}^{-3}$. The ion temperature and product $Z T_{e}$ were measured using Thomson scattering to be $T_{i} \sim 60 \mathrm{eV}$ and $Z T_{e} \sim 150$ to $200 \mathrm{eV}$. The measured plasma rotation velocity is $\sim 60$ $\mathrm{kms}^{-1}$ and it is supersonic (Mach number $\sim 2$ ). In addition both the Reynolds number $\left(\sim 10^{5}\right)$ and magnetic Reynolds number $(10-100)$ are large enough for viscous and resistive effects to be negligible on the large scale of the flow.

Although these results are preliminary, we have demonstrated that this experimental platform can be used to generate rotating, compressible, ideal magneto-hydrodynamic flows under conditions that are potentially well suited to study certain aspects of accretion disk physics. In particular, it was seen that the flow rotates differentially and should therefore allow for studying sheared, compressible instabilities in a curved geometry. This is different from plane-parallel sheared flows where, for example, the equilibrium conditions do not involve the centrifugal force.

In terms of time-scales, the total duration of our experiments corresponds to approximately one full revolution of the plasma; this is very different from astrophysical disks where the matter rotates in a quasi-steady equilibrium slowly making its way towards the centre. Therefore the experimental system in its present form will be best suited to investigating the development of fast instabilities with growth rates the same order or less than the rotation period. In addition the interaction at the boundary between the azimuthally localised incoming streams and the rotating flow will act as a driving force that could lead to rapid development of instabilities and turbulent motions. In an astrophysical context similar configurations are expected to be present in the accretion processes in double-star configurations; for example in cataclysmic binaries [11, 12, 13]. In general we estimate the Reynolds number to be large enough $\left(R e \sim 10^{5}\right)$ for viscous effects to be neglected and for any turbulent motion to be sustained.

Magnetic fields are also important in accretion disks, where the transport of angular momentum is thought to be the result of turbulence generated by the magnetorotational instability. We estimate that the magnetic Reynolds number in our experiments is sufficiently high
$\left(R e_{m} \sim 10-100\right)$ that the magnetic field is well coupled to the plasma to have an effect on its dynamics. As shown in other wire array z-pinch experiments, the magnetic field is advected by the plasma streams $[14,15,16,17]$ and we expect that a predominantly toroidal magnetic field will be present in the rotating plasma. However the strength of this field is as yet poorly constrained. Numerical simulations [2] indicate that the plasma beta (ratio of thermal to magnetic pressures) in the incoming plasma streams exceeds unity by the order of a few and similar values are expected in the rotating flow. Measurement of the magnetic field strength in the flow will be attempted in future experiments.

\section{Acknowledgements}

This work was supported in part by EPSRC Grant No. EP/G001324/1 and by DOE cooperative agreements No. DE-F03-02NA00057 and No. DE-SC-0001063

\section{References}

[1] S. A. Balbus, J. F. Hawley, A Powerful Local Shear Instability in Weakly Magnetized Disks I. Linear Analysis, Astrophys. J. 376 (1991) 214-222.

[2] M. Bocchi, J. P. Chittenden, S. V. Lebedev, G. N. Hall, M. Bennett, A. Frank, E. G. Blackman, Numerical simulations of Z-pinch experiments to create supersonic differentiallyrotating plasma flows, High Energy Density Phys. 9 (1) (2013) 108-111. doi:10.1016/j.hedp.2012.12.001.

URL http://linkinghub.elsevier.com/retrieve/pii/ $\mathrm{S} 1574181812001346$

[3] M. Bocchi, B. Ummels, J. P. Chittenden, S. V. Lebedev, A. Frank, E. G. Blackman, Numerical Simulations of ZPinch Experiments To Create Supersonic Differentially Rotating Plasma Flows, Astrophys. J. 767 (1) (2013) 84. doi: 10.1088/0004-637X/767/1/84.

URL http://stacks.iop.org/0004-637X/767/i=1/a=84?key= crossref. 863a4d9c52557ea8ccf cede2334bfd6c

[4] D. J. Ampleford, S. V. Lebedev, A. Ciardi, S. N. Bland, S. C. Bott, G. N. Hall, N. Naz, C. Jennings, M. Sherlock, J. P. Chittenden, J. Palmer, A. Frank, E. G. Blackman, Supersonic Radiatively Cooled Rotating Flows and Jets in the Laboratory, Phys. Rev. Lett. 100 (3) (2008) 035001. doi: 10.1103/PhysRevLett. 100.035001.

URL http://link.aps.org/doi/10.1103/PhysRevLett.100. 035001

[5] D. D. Ryutov, Using intense lasers to simulate aspects of accretion discs and outflows in astrophysics, Astrophys. Space Sci. 336 (1) (2010) 21-26. doi:10.1007/s10509-010-0558-9. URL http ://link. springer.com/10.1007/s10509-010-0558-9

[6] S. V. Lebedev, F. N. Beg, S. N. Bland, J. P. Chittenden, a. E. Dangor, M. G. Haines, K. H. Kwek, S. a. Pikuz, T. a. Shelkovenko, Effect of discrete wires on the implosion dynamics of wire array Z pinches, Phys. Plasmas 8 (8) (2001) 3734. doi: 10.1063/1.1385373

URL http://scitation.aip.org/content/aip/journal/pop/ 8/8/10.1063/1.1385373

[7] G. F. Swadling, S. V. Lebedev, N. Niasse, J. P. Chittenden, G. N. Hall, F. Suzuki-Vidal, G. C. Burdiak, A. J. HarveyThompson, S. N. Bland, P. de Grouchy, E. Khoory, L. Pickworth, J. Skidmore, L. Suttle, Oblique shock structures formed during the ablation phase of aluminium wire array z-pinches, Phys. Plasmas 20 (2) (2013) 022705. doi:10.1063/1.4790520. URL http://scitation.aip.org/content/aip/journal/pop/ 20/2/10.1063/1.4790520 
[8] A. J. Harvey-Thompson, S. V. Lebedev, S. Patankar, S. N. Bland, G. C. Burdiak, J. P. Chittenden, A. Colaitis, P. de Grouchy, G. N. Hall, E. Khoory, M. Hohenberger, L. Pickworth, F. Suzuki-Vidal, R. A. Smith, J. Skidmore, L. Suttle, G. F. Swadling, Optical Thomson scattering measurements of cylindrical wire array parameters, Phys. Plasmas 19 (5) (2012) 056303. doi:10.1063/1.3694671.

URL http://scitation.aip.org/content/aip/journal/pop/ 19/5/10.1063/1.3694671

[9] D. H. Froula, S. H. Glenzer, N. C. Luhmann, J. Sheffield, Plasma Scattering of Electromagnetic Radiation, 1st Edition, 2011.

[10] L. D. Landau, E. M. Lifshitz, Fluid Mechanics, 2nd Edition, 1987.

[11] P. J. Armitage, M. Livio, Hydrodynamics of the Stream-Disk Impact in Interacting Binaries, Astrophys. J. 493 (2) (1998) 898-908. doi:10.1086/305149.

[12] S. Kunze, R. Speith, F. V. Hessman, Substantial stream-disc overflow found in three-dimensional SPH simulations of cataclysmic variables, Mon. Not. R. Astron. Soc. 322 (3) (2001) 499-514. doi:10.1046/j.1365-8711.2001.04057.x.

[13] M. R. Truss, Accretion disc dynamics in extreme mass ratio compact binaries, Mon. Not. R. Astron. Soc. 376 (1) (2007) 89-97. doi:10.1111/j.1365-2966.2007.11437.x.

[14] V. V. Aleksandrov, V. A. Barsuk, E. V. Grabovski, A. N. Gritsuk, G. G. Zukakishvili, S. F. Medovshchikov, K. N. Mitrofanov, G. M. Oleinik, P. V. Sasorov, Studies of penetration of the magnetic field into electrically imploded loads in the Angara-5-1 facility, Plasma Phys. Reports 35 (3) (2009) 200221. doi:10.1134/S1063780x09030039.

[15] J. Greenly, M. Martin, I. Blesener, D. Chalenski, P. Knapp, R. McBride, The Role of Flux Advection in the Development of the Ablation Streams and Precursors of Wire Array Z-pinches, AIP Conf. Proc. 1088 (2009) 53-56.

[16] G. F. Swadling, S. V. Lebedev, A. J. Harvey-Thompson, W. Rozmus, G. C. Burdiak, L. Suttle, S. Patankar, R. a. Smith, M. Bennett, G. N. Hall, F. Suzuki-Vidal, J. Yuan, Interpenetration, Deflection, and Stagnation of Cylindrically Convergent Magnetized Supersonic Tungsten Plasma Flows, Phys. Rev. Lett. 113 (3) (2014) 035003. doi:10.1103/PhysRevLett.113.035003.

URL http://link.aps.org/doi/10.1103/PhysRevLett.113. 035003

[17] G. F. Swadling, S. V. Lebedev, G. N. Hall, S. Patankar, N. H. Stewart, R. a. Smith, a. J. Harvey-Thompson, G. C. Burdiak, P. de Grouchy, J. Skidmore, L. Suttle, F. Suzuki-Vidal, S. N. Bland, K. H. Kwek, L. Pickworth, M. Bennett, J. D. Hare, W. Rozmus, J. Yuan, Diagnosing collisions of magnetized, high energy density plasma flows using a combination of collective Thomson scattering, Faraday rotation, and interferometry (invited)a), Rev. Sci. Instrum. 85 (11) (2014) 11E502. doi:10.1063/1.4890564.

URL http://scitation.aip.org/content/aip/journal/rsi/ $85 / 11 / 10.1063 / 1.4890564$ 